\title{
Die Gaben fliessen nicht mehr - zum Verhältnis von singulärer und gesellschaftlich eingebundener psychischer Struktur ${ }^{1}$
}

Dagmar Ambass

Zusammenfassung: Ausgehend von einer Falldarstellung über eine Schwarzafrikanerin, die nach ihrer Heirat mit einem Schweizer Mann die Symptomatik einer Psychose zeigt, wird untersucht, ob das Herausfallen aus gesellschaftlichen Bezügen, die in afrikanischen Gesellschaften von einem gegenseitigen Geben und Nehmen geprägt sind, zum Ausbruch der Psychose geführt haben könnte. Dazu werden Lacans Konzept des Sinthom und eine ethnopsychoanalytische Analyse von gesellschaftlichen Strukturen und Übergangsprozessen herangezogen.

Schlüsselwörter: Signifikantentheorie, Name-des-Vaters, Sinthom, Vatermetapher, Verwerfung, Verleugnung, Verdrängung, Mangel, Kastration, Geniessen, Objekt a, Klinik des Objekt a, Formationen des Unbewussten, Formationen des Objekt a, delirierende Metapher, Matrilinearität, Matrifamilie, patriarchale Kernfamilie

\section{$1 \quad$ Falldarstellung}

Frau Streit ${ }^{2}$ verliess ihr Heimatland in Westafrika auf der Suche nach einem Auskommen. Und sie hatte Glück ("I was lucky"), denn sie begegnete einem Schweizer Mann, mit dem sie heute verheiratet ist. Ich lernte sie und ihren Mann kurz vor ihrem Austritt aus einer psychiatrischen Klinik kennen. Nach Meinung des Helfersystems benötigte sie Unterstützung bei der Betreuung der gemeinsamen Kinder und beim Führen des Haushalts. Bei unserer ersten Begegnung wirkte sie auf mich wie versteinert. Ich konnte ihr ein Lächeln abgewinnen, indem ich erzählte, dass ich vor vielen Jahren Ghana besucht hätte. Frau Streit hatte in Ghana Freunde, die ihr in schwierigen Zeiten beigestanden waren. Ich begleitete sie im Rahmen von psychoanalytischer Sozialarbeit über mehrere Jahre. Zunächst sprach sie kaum und antwortete einsilbig auf meine Fragen. Schliesslich schlug ich vor, dass wir gemeinsam backen könnten. Ich würde ihr meine Rezepte zeigen, von ihr 
würde ich gern etwas über die afrikanische Küche erfahren. Auf diesen Vorschlag ging Frau Streit ein, mit so viel Eifer, dass die Kinder, die sich gern beteiligt hätten, ausgeschlossen wurden. Das waren wichtige Angelegenheiten unter Erwachsenen, kein lustvolles Kneten für Kinder. Mit diesem Geben und Nehmen kam unsere Beziehung in Gang.

Die Beziehung zwischen den Eheleuten war konfliktreich. Nach und nach erfuhr ich etwas über ihre gemeinsame Geschichte. Kurz nach Frau Streits Ankunft in der Schweiz lernten sie sich an einer afrikanischen Veranstaltung kennen. Das erste gemeinsame Kind war schnell unterwegs. Herr Streit wünschte, dass es in der Schweiz aufwachsen könne. Dies war ein gewichtiger Grund dafür, dass er in eine Heirat mit Frau Streit einwilligte. Herrn Streits Herkunftsfamilie war mit dieser Heirat zunächst nicht einverstanden. Einige seiner Geschwister weigerten sich in den ersten Jahren ihrer Ehe, Frau Streit kennen zu lernen, was sie sehr verletzte. Es war ihr unverständlich, dass sie keinerlei Hilfe von ihren neuen Verwandten erwarten konnte. Schliesslich änderten zwei Schwägerinnen ihre Haltung. Sie bemühten sich, zu Frau Streit Kontakt herzustellen und wollten sie in der Haushaltsführung und Kindererziehung unterstützen. Frau Streit fühlte sich durch diese gut gemeinte Hilfe bevormundet und nicht als erfahrene Hausfrau und Mutter respektiert. Herr Streit wurde zwischen den Konflikten der Frauen aufgerieben und wusste nicht, wie er schlichten sollte. Verständnis hatte er für beide Seiten. Die Erwartung seiner Frau, dass er für ihre Position in der Familie einstehe, konnte er nur teilweise erfüllen.

Ein Grund dafür, dass Frau Streit fern ihrer Heimat Geld verdienen wollte, waren ihre beiden älteren Kinder, die in Afrika lebten und denen sie eine mit hohen Kosten verbundene Ausbildung ermöglichen wollte. Dies ist ihr trotz schwieriger Lebensumstände bis heute gelungen. Die Verpflichtung diesen beiden Kindern gegenüber führte zwar immer wieder zu Konflikten mit Herrn Streit und seiner Herkunftsfamilie, jedoch gelang es Frau Streit stets von Neuem, wenn nötig, die Unterstützung ihres Mannes zu mobilisieren. Mithilfe der lokalen afrikanischen Gemeinschaft in einer Schweizer Grossstadt, wo die Familie lebt, - und dabei handelte es sich um zufällige Begegnungen und nicht um bewährte, verlässliche Beziehungen - fand Frau Streit sowohl Arbeit als auch Hilfe bei der Kinderbetreuung. Sie bewies Durchhaltevermögen über viele Jahre beim gleichen Arbeitgeber - bis die psychotischen Krisen einsetzten und schliesslich zum Verlust der Stelle führten.

Zunächst entwickelte Frau Streit einen Eifersuchtswahn. Sie dachte, eine andere Frau wolle ihr den Mann streitig machen. Schliesslich litt sie unter der Vorstellung, dass an ihren Kleidern, Schuhen und Schmuckstücken manipuliert werde. Diese Vorstellung blieb auch in Phasen, in denen Frau Streit nicht akut 
psychotisch war, erhalten. In Zusammenhang mit den Manipulationen an ihrer Kleidung kamen wir auf die Beziehung zu den Schwägerinnen zu sprechen. Frau Streit vermutete, dass eine von ihnen glaube, sie wolle sie verhexen. Dazu führte sie folgendes Beispiel an: Als die Schwägerin zu Besuch kam, nahm sie von Frau Streit kein Wasser an. Sie holte sich selbst ein Glas vom Wasserhahn. Indirekt brachte Frau Streit die Vermutung ins Spiel, dass die Verwandten ihres Mannes hinter den Manipulationen an den Kleidern steckten und dass Hexerei im Spiel sei. Wenn die Psychose akut wurde, lösten die Wahnvorstellungen bei Frau Streit massive Ängste und Aggressionen aus, die sich immer gegen ihren Mann richteten. Diese führten mehrmals zu einem fürsorglichen Freiheitsentzug in einer psychiatrischen Klinik. Sie war unglücklich während der Psychiatrieaufenthalte und litt unter den Nebenwirkungen der Medikamente, liess aber beides über sich ergehen. Ich gewann den Eindruck, dass sie sich selbst «opferte», um ihre Pflicht gegenüber den Kindern und anderen Verwandten in Afrika zu erfüllen. Denn sie hielt stets an der Ehe mit Herrn Streit fest, auch wenn sie sich unverstanden und schlecht behandelt fühlte. Frau Streit hatte klare Einsichten in die Unlösbarkeit einiger Konflikte, die sich in dieser binationalen Partnerschaft auftaten. Sie war sich bewusst, dass Afrika «ein Fass ohne Boden» ist, dass sich Herr Streit als reicher weisser Mann in ihrem Heimatdorf nicht sehen lassen kann, weil dort unerfüllbare Mitgiftforderungen an ihn herangetragen würden. Auch sie selbst kam bei ihren Besuchen in der Heimat in Bedrängnis und musste sich am Ende sogar vor Hilfesuchenden verstecken, obwohl sie mit vollen Taschen angereist war.

Meine Aufgabe bestand im Strukturieren innerhalb der Paarbeziehung. Mit dem Ehepaar diskutierte ich im Detail, wer für welche Angelegenheiten zuständig war und wie sich kleine Ärgernisse wie z. B. Reissäcke in der Küche durch Kompromisse aus der Welt schaffen liessen. Wir arbeiteten an der feinen Grenze zwischen persönlichen Eigenarten resp. Gewohnheiten, die der Partner oder die Partnerin respektieren musste, undWünschen und Bedürfnissen, die zum Wohl der gesamten Familie zurückgestellt werden mussten. Ein besonderes Augenmerk galt den Kindern, die ausserfamiliäre Unterstützung benötigten, da sie in Anbetracht der Paarkonflikte und der psychischen Erkrankung der Mutter vernachlässigt zu werden drohten. Solange ich als strukturierende Dritte real präsent war, nahmen die Paarkonflikte ab und Frau Streit konnte sich stabilisieren. Ihr Ziel war es, wieder im Arbeitsleben Fuss zu fassen. Ihr eigener Verdienst war für sie wichtig, um sich einerseits ihre eigenen Bedürfnisse nach Kleidern, Schuhen und Schmuck, nach Attributen derWeiblichkeit, erfüllen zu können und andererseits den Ansprüchen der Verwandten in Afrika gerecht zu werden. Mehrmals kam es zu erneuten psy- 
chotischen Krisen in Verbindung mit längeren ferienbedingten Unterbrechungen der Begleitung. Mit dem chronisch werdenden Krankheitsverlauf entstand ein Helfernetz, welches sich bemühte, eine tragende Struktur zu errichten, um die akut psychotischen Zustände abzuwenden. Dies gelang einerseits durch die Suche nach einer medikamentösen Behandlung mit erträglicheren Nebenwirkungen (Reduktion der Schlafstörungen), andererseits durch langfristige, tragende Beziehungen, beispielsweise bei der psychiatrischen Versorgung, der Betreuung der Kinder und im Rahmen eines Beschäftigungsprogramms. Unser Ziel war es, dass Frau Streit die im Aussen repräsentativ errichteten Strukturen internalisieren und somit mehr psychische Stabilität erlangen würde. Im Folgenden möchte ich einen Ansatz zum Verständnis der Krankheit und der psychischen Dekompensation entwickeln.

Durch die Erzählung eines Erlebnisses konnte mir Frau Streit ihr Dilemma begreiflich machen. Diese Erzählung kann vermutlich als Paradigma für die Funktion des Austausches in afrikanischen Gesellschaften gelten und auch zum Verständnis seiner Funktion in Bezug auf die singulären psychischen Strukturen beitragen: Frau Streit war unterwegs in der Stadt, in der sie lebte, als sie plötzlich in den Gängen der Untergrundbahn laute Stimmen hörte. Ein heftiger Streit wurde in ihrem heimatlichen afrikanischen Dialekt ausgetragen. Sie näherte sich einem Paar mit Kind, sprach es in ihrer Muttersprache an und wollte den Grund der Auseinandersetzung erfahren. Der Mann erklärte, dass sie in Spanien lebten und verheiratet seien. Er habe von einer Schwester ${ }^{3}$ eine Einladung in die Schweiz erhalten. Seiner Frau habe er ausdrücklich gesagt, dass die Einladung nur für ihn gelte und dass er auch nicht über genügend Mittel verfüge, um für ihre Fahrkarte aufzukommen. Schliesslich sei ihm seine Frau mit dem Kind nachgereist - mit einem Einwegticket. Nun habe sie kein Geld, um die Rückreise zu bezahlen und er sei nicht bereit, diese Kosten zu übernehmen. Frau Streit antwortete, sie habe leider auch nicht genügend Geld für die Reisekosten, sehe aber, dass der kleine Junge hungrig sei und könne den Eltern CHF 20.00 geben, damit sie im McDonalds Essen kaufen könnten. Daraufhin bedankte sich der Mann und sagte: «Schwester, ich wollte das Rückreiseticket nicht bezahlen, aber nun, da du so freundlich warst und uns CHF 20.00 gegeben hast, damit wir unseren Sohn nähren können, werde ich die Kosten übernehmen».

Mit ihrer Gabe hat Frau Streit den Fluss der Güter in Gang gesetzt und ermöglicht, dass auch bei dem Paar wieder ein Geben und Nehmen stattfinden konnte. Dabei hat sie selbst einen Einsatz geleistet und einen realen Verlust in Kauf genommen. Offenbar liegt der zentrale Punkt beim Geben und Nehmen nicht 
darin, einen Ausgleich zu schaffen. Ein Verlust kann auch mit einem Gewinn verbunden sein, möglicherweise, indem durch den Verlust für ein Geniessen bezahlt wird. Dieses Geben und Nehmen könnte auch für Frau Streit die Grundlage jeglicher Beziehungen sein. Ich vermute, dass sie aus tragenden Bezügen herausgefallen ist, in denen ein realer Austausch die psychische Strukturierung stützt resp. als Sinthom wirkt. ${ }^{4}$ Der Fluss der Signifikanten, der Austausch von Wörtern allein, reicht nicht aus, um Beziehungen zu bekräftigen. Er schützt nicht genügend vor der Bedrohung durch schädigende Geister, vor Neid, Eifersucht und - im weitesten Sinn - Loyalitätskonflikten. Ein weiterer Aspekt erscheint mir in diesem Zusammenhang bedeutsam: Wie viele andere schwarzafrikanische Christen verbrachte Frau Streit Stunden damit, DVDs mit Bekenntnissen von Gläubigen, die Wunder erlebt hatten, anzusehen und CDs mit Gospels zu hören, in denen die gleichen Glaubenssätze wieder und wieder beschworen werden, sowie freikirchliche Gottesdienste zu besuchen. Es reicht nicht aus, an Gott zu glauben. Insbesondere reicht es nicht aus, einmal zu sagen oder gar nur zu denken, «ich glaube an Gott» und damit die eigene Beziehung zu Gott zu klären. Die Religion muss in einem fort praktiziert werden.

Können diese beiden Elemente sowohl als Funktion der psychischen Struktur resp. als Symptome des Subjekts als auch konstitutiv für das Funktionieren einer gesellschaftlichen Gruppe verstanden werden? Um dieser Frage nachzugehen, nehme ich einen Umweg über die strukturale Psychoanalyse und die strukturale Sozialanthropologie.

\section{Vom Namen-des-Vaters zum Sinthom bei Lacan}

Aus einer phänomenologisch-psychiatrischen Sicht leidet Frau Streit an einer paranoiden Psychose, die sich in einem Verfolgungswahn mit Stimmen hören äussert, evtl. sogar an einer Schizophrenie, da körperliche Sensationen, u. a. Brennen der Beine und ein vergrösserter Bauch, als ob sie schwanger wäre, hinzukommen. Wie lässt sich die Störung von Frau Streit aus einer strukturellpsychoanalytischen Perspektive einordnen?

Wie PeterWidmer (2007) vermutet, ist Sigmund Freud der Entdeckung einer Theorie der Signifikanten sehr nahe gekommen. In seinem Aufsatz «Paraphrenie ein vergessenes Konzept Freuds» geht er der Entwicklung der Unterscheidung von Neurose und Psychose anhand des Begriffs der Paraphrenie nach. Insbesondere mit der Unterscheidung von Wortvorstellungen und Sachvorstellungen stand Freud kurz vor der Entdeckung einer Signifikantentheorie. Jacques Lacan entwickelte daran anknüpfend die Theorie der Signifikantenstruktur ${ }^{5}$ und nennt zwei 
Bezugspunkte, die es erlauben, psychische Störungen in einem strukturellen Ordnungssystem zu erfassen, nämlich den Bezug zum anderen ${ }^{6}$ und den Bezug zum Mangel resp. zum Objekt $a^{7}$. Um seine Theorie der psychischen Strukturen darzulegen, orientiere ich mich an dem Aufsatz «Eine kurze Einführung in die Theorie der klinischen Strukturen» von Christoph Keul (2007). Zunächst sei Sciacchitanos Hinweis erwähnt, dass ein Ordnungssystem nie die Gesamtmenge an psychischen Störungen erfassen kann, sondern lediglich eine Teilmenge, und die klassifizierbaren psychischen Störungen immer nur partiell klassifizierbar sind, d.h. nur in Bezug auf einige definierbare Merkmale. Grund für diese nicht vollständige Klassifizierbarkeit ist das Reale, der Rest, der nicht in der Ordnung der Signifikanten erscheint und sich jeglicher Systematisierung entzieht.

Die Lacansche Diagnostik orientiert sich an der vom Subjekt gewählten «Position, die es im Durchlauf des Ödipus in seiner Beziehung zum anderen gegenüber dem Mangel - anders formuliert: in Hinblick auf das Geniessen (Nicht-Anerkennung des Mangels) bzw. die Kastration (Anerkennung des Mangels) - einnimmt» (Keul 2007: 20). Der frühe Lacan entwickelt zur Beschreibung dieses Vorgangs und zur Beschreibung der daraus resultierenden Strukturen die Vatermetapher, welche aus einer entwicklungspsychologischen Perspektive folgendermassen gelesen werden kann: Am Anfang hat das Kind vor allem mit der Mutter zu tun. Bald stellen sich Schwierigkeiten in der Mutter-Kind-Beziehung ein, die mit dem Mangel zu tun haben. Die Mutter ist offensichtlich nicht immer im passenden Augenblick da, wenn sich die Bedürfnisse des Kindes bemerkbar machen, und sie scheint auch nicht allein für das Kind da zu sein. Sie hat andere Interessen und begehrt noch etwas anderes ausser ihrem Kind. Indem die Mutter dies zu erkennen gibt, beginnt das Kind mit diesem unbekannten anderen zu rivalisieren. Es möchte allein den Platz des begehrten Objekts bei der Mutter einnehmen. Diese Position räumt ihm die Mutter immer weniger ein. Je heftiger das Kind in diese Position drängt, umso mehr wird es früher oder später entweder vom Vater selbst oder häufiger von der Mutter im Namen des Vaters zurückgewiesen. Das Kind übernimmt implizit von der Mutter diese zentrale Bezugsgrösse, die der Name-des-Vaters offensichtlich auch für die Mutter darstellt. Somit gewinnt er im Unbewussten des Kindes eine Schlüsselstellung, die zuvor dem Begehren der Mutter zukam. Diese Ersetzung des Begehrens der Mutter durch den Namen-desVaters (Vatermetapher) erfolgt also in einem Moment, in dem es um die Etablierung des Gesetzes geht, genauer gesagt des Inzesttabus, das sowohl dem Kind wie der Mutter ein Verbot und damit einen Verlust auferlegt. Somit bildet die Aufnahme dieses Signifikanten ins Unbewusste des Subjekts den Kern der symbolischen

Psychosen - Psychoanalytische Perspektiven 
Ordnung (Keul 2007: 21). Wichtig ist, dass die Aufnahme des Namens-des-Vaters in zwei Schritten erfolgt: Zunächst muss der Mangel der Mutter anerkannt werden sowie auch die Unmöglichkeit, dass das Kind diesen Mangel stillen kann. Im zweiten Schritt muss das Subjekt erkennen, dass es auch selbst dem Mangel unterliegt. Weder darf das Kind die Mutter ganz für sich beanspruchen noch die Mutter das Kind. Darin liegt die Bedeutung des «Nein», das im nom/non du père (Nein/Namedes-Vaters) anklingt. Es gilt sowohl der Mutter als auch dem Kind. In Abhängigkeit davon, ob diese Schritte gar nicht, teilweise oder ganz vollzogen worden sind, können vier Positionierungen des Subjekts gegenüber dem Mangel in der Beziehung zum anderen unterschieden werden. Drei davon sind klinisch relevant:

, Der Mangel wird nicht anerkannt, sondern gänzlich negiert, d.h. er wird nicht symbolisiert. Damit wird der Eintritt in den Ödipus verfehlt. Lacan spricht von der Verwerfung des Signifikanten des Namens-desVaters. Dies bedingt die Struktur der Psychose. Der Psychotiker nimmt die Konsequenzen des Eintritts in die Sprachordnung nicht auf sich. Er akzeptiert das Verbot des Geniessens nicht. Damit ist er dem Geniessen ungeschützt ausgesetzt, z. B. in Form von Halluzinationen. Die Klinik der Psychose ist geprägt durch den Einbruch des Geniessens oder mit anderen Worten durch Phänomene der Ordnung des Realen. Freudianisch gesprochen hat die Urverdrängung nicht stattgefunden. $\mathrm{Zu}$ den charakteristischen Symptomen gehören Störungen des Sprechens, sei es, dass das Sprechen zu starr wird (Paranoia) oder zu locker bis hin zur völligen Auflösung der Satzstruktur (Schizophrenie). Ausserdem kann z. B. das Fehlen einer Symbolisierung der An- und Abwesenheit des anderen zum Phänomen des Verfolgungswahns führen. ${ }^{8}$ Diese Symptomatik finden wir auch bei Frau Streit. Die Stimmen, die sie hört, kündigen ihr beispielsweise die Ankunft von Bekannten aus ihrer Heimat oder von Freunden an, die auf ihrem Lebensweg eine Zeitlang von Bedeutung waren. Teilweise sind die Stimmen freundlich, teilweise nehmen sie bedrohliche Formen an, indem sie sie selbst oder ihre Kinder mit dem Tod bedrohen. Keul merkt jedoch an, dass das Fehlen der Urverdrängung nicht immer zur Psychose führt, «denn es kann sein, dass ein Subjekt ein Symptom dazu benutzen kann, die nötige Trennung vom ersten anderen (der Mutter) voranzubringen und damit eine Anerkennung des Inzestverbots und eine Symbolisierung des Mangels zu leisten. Wenn ein Symptom diese Funktion, die in der Neurose zunächst einmal dem Namen-des-Vaters zukommt, erfüllen kann, nennt Lacan es ein Sinthom»(Keul, 2007: 23). 
, Wenn der erste Schritt gelungen ist und das Subjekt den Namen-des-Vaters ins Unbewusste aufgenommen hat, kommt es darauf an, ob es auch die Konsequenzen dieser Entscheidung auf sich nimmt. Es kann sein, dass das Subjekt ein Phantasma ${ }^{9}$ ausbildet, welches das Begehren nach dem Ausgleich des Mangels und der (Wieder-)Herstellung der Einheit stützt. Diese Form der partiellen Anerkennung des Mangels gibt es in zwei grundsätzlich unterscheidbaren Varianten. Die erste entspricht der Perversion. Der Perverse sieht den Mangel nur auf der Seite des anderen und fühlt sich berufen, diesen Mangel zu beheben und ihm Befriedigung zu verschaffen (Phallus sein). Er ist identifiziert mit dem Objekt a, oft in der fixierten Form des Fetischs. Lacan spricht von der Abwehrform der Verleugnung des Signifikanten des Namens-des-Vaters. Die Transformation des imaginären in den symbolischen Phallus (Identifizierung mit dem Namen-des-Vaters) ist für das Subjekt nicht erfolgt.

, Die zweite Variante der partiellen Anerkennung des Mangels entspricht der Neurose. Das Subjekt hat anerkannt, dass es am Mangel leidet, d.h. der Phallus als Signifikant des Mangels ist im Unbewussten eingeschrieben. Die Transformation des imaginären in den symbolischen Phallus ist zumindest teilweise erfolgt. Das Inzesttabu wird akzeptiert. DerVerzicht auf die Mutter ist geleistet. In seiner Phantasie malt sich der Neurotiker jedoch aus, dass er in einem anderen Menschen doch noch das Objekt findet, das seinen Mangel beheben kann (Phallus haben). Der Mangel befindet sich auf der Seite des Subjekts. Auf der Seite des anderen ist das Objekt, das dem Anschein nach den Mangel beheben kann. Die zur Neurose gehörige Abwehrform ist die Verdrängung. Anders als der Perverse will der Neurotiker seine Phantasie nicht realisieren. Die Abwehr gegen das Geniessen ist viel stärker. Er fürchtet sich sogar vor der Realisierung seines Phantasmas. Nur im Symptom überschreitet er die Grenzen des Lustprinzips und erreicht ein (schmerzhaftes) Geniessen (z. B. in Form des Wiederholungszwangs). Der Neurotiker bleibt in einer imaginären Interpretation der Kastration verhaftet. Er glaubt, sein Mangel entstehe aufgrund des Anspruchs des anderen an ihn, den er dafür verantwortlich macht, dass ihm das begehrte Objekt vorenthalten wird. Er verkennt, dass es nicht in der Macht des anderen steht, ihm die Erfahrung des Mangels zu ersparen.

, Bei der vierten Form, die sehr selten vorkommt, ist auch die Symbolsierung des Mangels im anderen vollzogen worden. 
Die psychische Struktur resultiert aus der «erzwungenen Wahl», die das Subjekt in Bezug auf die Positionierung gegenüber dem Mangel trifft. Es resultiert in der Regel entweder die neurotische, psychotische oder perverse Struktur. Damit ist ein grosser Teil der klinischen Strukturen abgedeckt, aber sicher nicht alle. Klassifizieren im strukturalistischen Sinn lässt sich nur, was zur Ordnung des Symbolischen oder des Imaginären gehört. Die Lacansche Psychoanalyse orientiert sich am Subjekt des Unbewussten, dessen Bedingung die Sprache ist, und erarbeitet ihre Unterscheidungen in der Ordnung des Symbolischen, denn der Mangel wird ja gerade durch die Sprache resp. durch die Differenz zwischen Signifikat und Signifikant eingeführt. Lacan beschreibt den Prozess der Subjektwerdung, der mit der erzwungenen Wahl einhergeht, über die Jahre auf unterschiedliche Weise: zunächst mit der Vatermetapher, später mit den Prozessen von Alienation und Separation und schliesslich mit der Theorie des Sinthom. In der Klinik werden die in der symbolischen Ordnung begründbaren Klassen gestört durch die Einwirkung des «nicht klassifizierbaren Rests» resp. des Objekts a. Dazu gehören alle Phänomene, die von der Ordnung des Realen sind wie z. B. Halluzinationen, passages à l'acte (Agieren, statt Symbolisieren), psychosomatische Phänomene usw. Aufgrund der Einwirkung des Objekts a können die Kriterien, nach denen die Strukturen unterschieden werden, schwer erkennbar sein. Zur Beschreibung der akuten Einwirkung des Objekts a wurden verschiedene Begriffe eingeführt wie die Klinik des Objekts a (Keul 2007: 28) oder Formationen des Objekts a (Nasio 1987 zit. n. Keul 2007: 29). Verhaeghe spricht in Anlehnung an Freuds Begriff der Aktualneurose allgemeiner von Aktualpathologien (2004 zit n. Keul 2007: 29). Was zum Zustand der Aktualpathologie gehört, lässt sich zunächst nicht klassifizieren. Wenn durch die Bindung des Geniessens und die Symbolisierung des Mangels in der Therapie der aktualpathologische in einen psychopathologischen Zustand überführt werden konnte, ist im psychopathologischen Zustand eine Klassifizierung oft wieder möglich (Keul 2007: 17-18). Aufgrund des Vorkommens nicht klassifizierbarer Fälle bleibt auch für die Psychoanalyse weiterhin die beschreibende Diagnostik wichtig.

Auch wenn die Theorie der Signifikanten nur vier «existentiale Strukturen» (A. Juranville [siehe dazu S. 7ff. in diesem Text]) abzuleiten erlaubt, es kann weitere Strukturen geben, die «eine Klasse für sich» sind, wie es Sciacchitano formuliert. Die topologische Deduktion $\left.{ }^{10}\right]$ der klinischen Strukturen [Sinthom] erlaubt also - im Gegensatz zur signifikantentheoretischen Ableitung [Name-des-Vaters] - auch die Möglichkeit der Existenz weiterer (singulärer) Struktu- 
ren zu begründen. (...) Die ödipal begründbaren Strukturen wären dann ein Spezialfall dieses allgemeineren Modus der Strukturbildung; sie bildeten eine Teilmenge, die dadurch charakterisiert wäre, dass in ihr das Sinthom gerade der Name-des-Vaters ist. Der Namedes-Vaters als Spezialfall des Sinthom führt zu den bekannten klinischen Strukturen, die aus dem Ödipuskomplex deduziert werden können. (Keul 2007:19)

Bei der Behandlung dieser Aktualpathologien ist es wichtig, dass der Therapeut immer auch den Mangel in der Übertragungsbeziehung bzw. die Grenzen seiner Behandlungsmöglichkeiten anerkennt. Dies kann beispielsweise durch ein vernetztes Arbeiten geschehen, wo einzelne Therapeuten oder Sozialarbeitende klar umgrenzte Aufgaben haben und bei darüber hinaus gehenden Erfordernissen auf den anderen verweisen. Die mangelnde Struktur im Psychischen resp. in der Beziehung zwischen dem Subjekt und dem anderen wird durch eine äussere Struktur ersetzt.

Keul vermutet, dass die Unterscheidung zwischen Formationen des Unbewussten und Formationen des Objekts $a$ in ähnlicher Weise schon von Freud getroffen worden ist, wenn er die Begriffe Aktualneurose und Psychoneurose verwendet. Er spricht von (sexual-)toxisch bedingter Aktualneurose, die einer analytisch orientierten Psychotherapie nicht zugänglich ist. Lacan präzisiert, dass es um die toxische Wirkung des Geniessens geht, d.h. «das Reale des Triebes», welches nicht über Signifikanten repräsentiert ist. Dagegen beinhalten die Psychoneurosen bereits eine «Verwörterung» der Trieb-Erfahrung. Es gibt Signifikanten, an die das Geniessen in einem gewissen Mass gebunden werden konnte. Auch Keul schlägt vor, diese Unterscheidung auf die Psychosen und die Perversionen auszudehnen und allgemein von aktualpathologischen und psychopathologischen Zuständen zu sprechen. In der Psychose kann beispielsweise zwischen einem gut ausgearbeiteten fixierten Wahn, einer delirierenden Metapher, wie Lacan (2005 zit. n. Keul 2009: 30) es nennt, und einem akut psychotischen Zustand unterschieden werden. Der aktualpathologische Zustand kann bei allen klinischen Strukturen ähnlich aussehen. Die Borderline-Pathologie entspricht in dieser Konzeptualisierung dem aktualpathologischen Zustand innerhalb einer neurotischen Struktur (Verhaeghe 2004 zit. n. Keul 2007: 30). In der Psychopathologie ist die Verwörterung teilweise geleistet, teilweise aber auch durch Abwehrmechanismen umgangen oder vermieden resp. aufgeschoben worden. Aufgrund der verschiedenen Abwehrmechanismen ergeben sich unterschiedliche psychopathologische Bilder für die verschiedenen klinischen Strukturen. «Mit Hilfe der Unterscheidung Aktualpathologie/ Psychopathologie einerseits und der Differenzierung der klinischen Strukturen 
innerhalb der Psychopathologie andererseits lässt sich jedenfalls auch die «neue Klinik oft gut beschreiben, ohne dabei die Freudsche Konzeption verlassen oder neue Strukturen erfinden zu müssen» (Keul 2007: 30).

Interessant in Bezug auf Frau Streit ist der Zeitpunkt des Ausbruchs der Psychose resp. der Aktualisierung der Psychose. Sie war fast 40 Jahre alt, hatte schon sehr viel früher Kinder geboren und war auch viele Jahre im Arbeitsleben aktiv gewesen. Wenn es sich bei ihrer Störung um eine Psychose im Lacanschen Sinn handelt, muss sie strukturell angelegt gewesen sein. Etwas, das als Ersatz des Namens-desVaters fungiert hat, muss weggefallen sein. Hier kann Lacans Begriff des Sinthom, den er in den 70er Jahren entwickelt hat, weiterhelfen. In dieser Schaffensperiode wird das Primat des Phallus als strukturierende Metapher durch das Primat des Sinthom ersetzt. Die Funktion des Sinthom ermöglicht es Lacan, auch diejenigen klinischen Phänomene zu beschreiben, die dadurch gekennzeichnet sind, dass der Name-des-Vaters verworfen ist. Die Vatermetapher resp. die Funktion des Phallus wird jetzt zu einem klinisch zwar wichtigen, aber doch nur speziellen Fall des Sinthom. Die Funktion des Sinthom besteht wie bei der Vatermetapher darin, nicht nur die Register des Realen, Imaginären und Symbolischen für ein spezifisches Subjekt miteinander zu verknüpfen, sondern auch seine Trennung vom ersten anderen zu ermöglichen. Ein Symptom kann in eine die Trennung fördernde Funktion transformiert werden und somit an die Stelle der fehlenden Vatermetapher treten oder eine ungenügend funktionierende ergänzen. Daraus können sich jenseits des Phallus neue, singuläre, subjektspezifische Varianten eines begrenzten Geniessens ergeben, die zugleich eine strukturierende Wirkung auf das Subjekt haben und ihm eine besondere Art der Sexuierung erlauben. Dies kann z. B. für die Klinik der Transsexuellen, besonders aber auch für die Klinik der Psychosen von Bedeutung sein. «Das Ende der Analyse kann dann als Identifikation mit dem «Sinthom` und als «das Wissen, was mit dem Sinthom zu tun sei` beschrieben werden» (Evans zit.n. Keul 2007: 31-32).

\section{$4 \quad$ Umweg über die strukturelle Sozialanthropologie}

Durch ihre Heirat mit einem gut situierten Schweizer, aus der zwei Kinder hervorgegangen sind, ist Frau Streit in ein Spannungsfeld zwischen ihrer Herkunftsund ihrer neuen Familie geraten. Sie bleibt weiterhin ihren Kindern in Afrika sowie weiteren Verwandten verpflichtet. Der eklatante Unterschied zwischen der Wohlstandsgesellschaft in der Schweiz und der Armut grosser Bevölkerungskreise im afrikanischen Herkunftsland sowie die Unkenntnis vieler in Afrika lebender Menschen des Sozialsystem der Schweiz und davon, wie viel Geld eine Familie 
benötigt, um alle finanziellen Verpflichtungen erfüllen zu können (Beiträge an soziale Sicherungssysteme, welche die verwandtschaftliche Solidarität weitgehend ersetzen) führen zu phantastischen Ansprüchen vonseiten der afrikanischen Verwandtschaft gegenüber der neugegründeten Familie Streit. Frau Streit ist sich sehr wohl bewusst, dass die Mittel ihres Ehemannes begrenzt sind. Sie kann sich aber der fordernden Haltung der Verwandten kaum erwehren und ist in einen Loyalitätskonflikt geraten.

Claude Levi-Strauss zieht in seinem Artikel «The Family» (1960) aufgrund eines Vergleichs und einer strukturellen Analyse unzähligerVerwandtschaftssysteme folgenden Schluss: Die Kernfamilie ist weder ein integrativer Teil einer grösseren sozialen Gruppe, noch bildet sie einen Grundbaustein der Gesellschaft. Vielmehr stehen die beiden Einheiten in einem nicht statischen, spannungsvollen Verhältnis zueinander. Die Gesellschaft ist für ihr Fortbestehen darauf angewiesen, dass sich neue Untergruppen bilden. Diese kleinen Einheiten bestehen jedoch immer nur auf Zeit. Sie müssen sich wieder auflösen, damit neue Einheiten entstehen können. Dies ist auch die Voraussetzung dafür, dass eine Gruppe wachsen kann. Dieser Widerspruch ist einzigartig für das menschliche Lebewesen. Er hat mit dem Antagonismus zwischen Natur und Kultur zu tun. Die Kultur kann sich nur weiter entwickeln, wenn soziale Gruppen solidarisch und in Abgrenzung zu anderen weiterbestehen und wachsen können. Gleichzeitig tut der Mensch gut daran, die Gesetze der Natur zu respektieren und neue Verbindungen in einem geregelten Mass zuzulassen. Levi-Strauss begreift die Verwandtschaftssysteme verschiedener Gesellschaften als mehr oder weniger gelungene Interpretationen diesesWiderspruchs. Kernfamilien sind sowohl die Bedingung als auch die Negation der Gesellschaft (Levi-Strauss 1960: 284-285).

In seinem Essay «Väter auf der Flucht» (Verhaeghe 2009: 87-160) wendet sich PaulVerhaeghe nach einer Demontage der ihn wenig überzeugenden Schrift Freuds «Totem und Tabu» ([1912] 1999) einer Neuinterpretation der Geschichte «Der Mann Moses und die monotheistische Religion» ([1939] 1999) zu. Während in der ersten Geschichte nur die Macht des Vaters über die Söhne thematisiert wird, geht es in der letzteren um die Beziehung zwischen Patriarchat und Matriarchat, mit dem Sohn als gemeinsamem Nenner. Der Mord am Urvater führt zur Einführung des Matriarchats und am Ende sind es die Söhne, die den patriarchalen Urvater wieder einsetzen. Verhaeghes Analyse zeigt, dass der Sohn den Vater einführt gegen eine mütterliche Macht, die als Bedrohung erfahren wurde. «Es handelt sich somit weniger um eine Furcht vor dem Vater als um ein Bedürfnis nach diesem Vater, um eine andere Gefahr zurückzubinden, eine Gefahr, die erst durch das Verschwinden des 
Vaters offenbar wurde und die geradewegs mit derWeiblichkeit in Zusammenhang steht. Mit anderen Worten: Der Vater ist das Symptom des Sohnes» (Verhaeghe 2009: 97). Für Verhaeghe ist der Wahrheitsgehalt des Mythos unerheblich. Mit Levi-Strauss besteht für ihn dessen Funktion darin, «eine kollektive Geschichte, die zu einer früheren psychischen Realität passt und die darauf folgende bestimmt» (Verhaeghe 2009: 97), in epischer Form darzustellen.

Ethnografische Forschungen zu verschiedenen Gesellschaften orientierten sich lange Zeit an zwei Fragestellungen, nämlich erstens worin bestehen die Regeln in Bezug auf das Sexualverhalten und zweitens wie sieht die Familienstruktur aus. Schliesslich wurde entdeckt, dass durchaus nicht immer, wie in der abendländischen Kultur, die Sexualität den am meisten regulierten Bereich darstellt. Bei primitiven Völkern ist das erste und wichtigste Element, das einer Regulierung unterworfen wird, das Essen. Sämtliche primordialen Regeln, die so genannten Tabus, betreffen das Essen und bestimmen bis ins Detail wer, was, wann und mit wem essen darf. Dies führt zu einer Segregation, die letztlich die Struktur der Sozialordnung des Stammes widerspiegelt. Daraus lässt sich ablesen, dass sich die Gruppen in der Regel nach dem gleichen Prinzip formieren: Innerhalb eines Klans steht auf der einen Seite die Gruppe der Mütter und Kinder und auf der anderen die der Jäger. Die Jungen wechseln ab einem bestimmten Alter, üblicherweise in der Pubertät, zur Gruppe der Männer. Der erwachsene Sohn isst nun nicht mehr gemeinsam mit der Gruppe der Frauen und Kinder. Zwischen den erwachsenen Frauen eines Klans und den erwachsenen Männern eines anderen Klans sind sexuelle Beziehungen erlaubt. Das Verwandtschaftsverhältnis läuft ausschliesslich über die Frauen und wird als matrilinear bezeichnet. Der Klan setzt sich somit aus zwei Untergruppen zusammen, auf der einen Seite den Frauen/Müttern, deren erwachsenen Töchtern und kleinen Kindern, auf der anderen Seite den Männern, das heisst den Söhnen, die alle zueinander sowie auch zu den erwachsenen Frauen Halbbrüder sind. Der Vater bzw. der Sexualpartner stammt aus einem anderen Klan und gehört nicht zum engsten Kreis. Insbesondere in Bezug auf die Verteilung der Lebensmittel läuft die Loyalität immer über die matrilineare Seite. Dem Sexualpartner und Vater, der einem anderen Klan angehört, gilt diese Loyalität nicht. Das Inzesttabu, das sich hier auf die Mitglieder des eigenen Klans bezieht, hat nichts gemein mit dem Inzesttabu, welches das über den Vater vermittelte ödipale Verbot in Bezug auf die Mutter darstellt. In dieser phylogenetisch ersten Lebensform gab es nicht nur keine Väter, sondern auch keine individuell zugeteilte Mutterschaft, es zählten nur die beiden Gemeinschaften des Klans, die der Frauen und die der Männer. 
Somit existierte auch kein Ichbegriff im abendländischen Sinn. Man war der Klan, Gruppe und Individuum waren miteinander vermischt.

Verhaeghe fragt, wie sich der Übergang ins patriarchale System vollzogen hat. In der Anthropologie wird die Matrifamilie als Übergangsform beschrieben: Diese funktioniert wie der ursprüngliche matrilineare Klan mit dem einzigen Unterschied, dass der männliche Sexualpartner aus einem anderen Klan bei den Mahlzeiten des Frauenklans dabei sein darf. Gleichzeitig mit der Paargründung kommt auch das Individuum ins Spiel. Daraus ergibt sich ein Konflikt mit der Loyalität, da einerseits die Loyalität an den Klan gebunden ist und andererseits die Sexual- und Essenspartner zwei verschiedenen Klans angehören. Nicht nur die Tatsache, dass über die Struktur des Klans hinausgegangen wird, führt zu einem Loyalitätskonflikt, sondern auch das gleichzeitige Entstehen der Begriffe von Besitz und Eigentum. Dieser Konflikt tritt beim männlichen Erstgeborenen besonders klar hervor: Das Mädchen bleibt in jedem Fall beim Klan der Mutter, in ihrer eigenen Untergruppe. Dagegen muss der Sohn zur Untergruppe der Männer/Söhne wechseln. In diesem Moment stellt sich die Frage, zu welcher, zu der des mütterlichen Klans, wie es früher üblich war, oder zu der des väterlichen Klans? Dies führt dazu, dass der erstgeborene Sohn im Übergangsprozess, der zwischen den beiden Systemen stattfindet, zum Zankapfel wird. Hier liegt auch der Ursprung eines Brauches, der bis an den Anfang der Zeitrechnung zurückreicht: die Opferung des Erstgeborenen, in der Regel stellvertretend an einem erstgeborenen männlichen Opfertier vollzogen. Auch die Mitgiftregelung stammt aus diesem Übergangsprozess. Dabei handelt es sich um eine Kompensation, die der väterliche Klan für die zu erwartenden Söhne entrichten muss. Möglicherweise gilt die Kompensation aber auch dem Geniessen, wenn auch die Frau in den Klan des Ehemannes/Vaters wechselt. Im Moment des Übergangs zur patriarchalen Familie erwirbt der Mann einen ausschliesslichen Anspruch auf das sexuelle Geniessen der Frau. ${ }^{11}$

Verhaeghe fährt fort, indem er mit der amerikanischen sozialistisch und feministisch orientierten Ethnologin Evelyn Reed den Ödipusmythos neu liest als Verarbeitung des Übergangs vom matrilinearen Klan zum Patriarchat. Das zentrale Thema stellt nicht der Inzest Mutter-Sohn dar. Vielmehr befindet sich Ödipus der Erstgeborene - genau an der Kreuzungsstelle der beiden Loyalitäten gegenüber dem mütterlichen Klan auf der einen und dem väterlichen auf der anderen Seite. Ödipus muss wählen, welchem Klan er angehören will. Dass dieses Thema wichtiger ist als der Inzest, zeigt sich darin, dass der Fluch, der über der Stadt liegt und die Strafe, die dem Protagonisten auferlegt wird, mit dem Vatermord in 
Verbindung stehen. Der übermässige Akzent, der auf den Inzest gelegt wird, stammt aus der postfreudianischen Auslegung (Verhaeghe 2009: 116-117). Am Ende kehrt Ödipus zu seiner Mutter Jokaste zurück, in den mütterlichen Schoss, den Klan seiner Mutter. Der Mord am Vater, unwissentlich begangen - und darin liegt die Tragik der Geschichte - hat zur Folge, dass das Erbe auf der mütterlichen Seite bleibt. Am Ende ist Kreon, der Bruder Jokastes, Thronfolger, nachdem Ödipus ins Exil gegangen ist. Daher muss der Ödipus als Geschichte, in der der Übergang vom matrilinearen Klan zum Patriarchat misslungen ist, gelesen werden. ${ }^{12}$ Verhaeghe folgert, dass der grundlegende Akt im Prozess der Menschwerdung im Vorgang der Trennung liegt. Er unterscheidet zwischen Autorität, Vaterschaft und Vaterfunktion. Autorität ist nicht mit Macht gleichzusetzen, sondern sie ist nur ein operatives Element im Vorgang der Trennung, gegen das sich die Macht sogar richten kann. Die Trennung stellt den Gegenpol zur Einheit dar.

Auf der Basis einer eingreifenden Autorität muss jedes werdende Subjekt seine ursprüngliche "Gruppe» - seine Mutter, seinen Klan, seine Kleinfamilie - verlassen, um in eine andere Gruppe einzutreten, um woanders etwas zu vollbringen.... Somit ist dieser Imperativ [bezogen auf die Kernfamilie: das Gebot, eine Person, die nicht der eigenen Kernfamilie angehört, zu heiraten] die Basis für die Kultur und Fortpflanzung des Menschen. In einer patriarchalischen Gesellschaft vollzieht sich die Trennung anhand der Vaterfigur. Nun sahen wir jedoch, dass die Kombination "Trennung/Vater» nicht zwangsläufig eine Notwendigkeit darstellt: Denn Trennungsvorschriften gab es bereits, als es noch gar nicht um den Vater oder die Vaterschaft ging. ... Das Verlassen einer Gruppe war gleichbedeutend mit dem Eintreten in eine andere, bis eine Art soziokulturelles perpetuum mobile erreicht war. Man sieht somit, dass die Bedeutung der Trennung selbst wesentlich ist, während das Verhältnis zur Vaterschaft und zum Patriarchat sekundären Charakter hat. Vom psychoanalytischen Standpunkt aus hat die Trennung noch eine weitere Dimension. Sie bewirkt nicht nur eine Distanzierung von einer bestimmten Gruppe oder Person, sondern sie schafft unmittelbar einen Abstand zwischen einem bestimmten Genuss und der Ureinheit, in der dieser Genuss erlebt wurde. Dadurch wird dem Bestreben, diesen Genuss wiederzuerlangen, eine allgemeine Beschränkung auferlegt, und gleichzeitig werden Austauschregeln eingeführt. Und zwar erfolgt das über jene Gruppe, die ihre Autorität in einer symbolischen Form schriftlich niederlegt, von mythischen Geschichten bis zu Gesetzestexten. (Verhaeghe [2004] 2009: 129-130) 


\section{Warum ist Frau Streit psychotisch geworden?}

In der Lacanschen Psychoanalyse wird der Ausbruch einer Psychose mit einer «wahren Begegnung» in Verbindung gebracht. Diese Begegnung verlangt dem Individuum einen folgenschweren Schritt ab, nämlich die ursprüngliche Gruppe (den Klan) bzw. in der Kernfamilie den ursprünglichen anderen (die Mutter) zu verlassen. Wenn man Verhaeghe in seiner Neuinterpretation des Ödipuskomplexes folgt, wird deutlich, dass die Heirat mit einem Schweizer, die für Frau Streit auch mit einem Namenswechsel verbunden war, in Bezug auf den Loyalitätskonflikt ungleich spannungsvoller war, als ihre erste Verbindung mit einem afrikanischen Mann, aus der zwei Kinder hervorgegangen sind, die jedoch Teil der matrilinearen Verwandtschaftsgruppe blieben. Möglicherweise waren die ersten beiden Geburten im Kontext der afrikanischen Gesellschaft nicht mit einem Schritt im Sinne einer wahren Begegnung verbunden. In unseren Gesprächen habe ich keinen Hinweis darauf gefunden, dass derVater ihrer ersten beiden Kinder eine bedeutsame Rolle gespielt hätte. Frau Streit war mit dem Vater dieser Kinder nicht verheiratet und trug vor ihrer Heirat den Namen ihres eigenen Vaters. Dagegen brachte sie die Heirat mit Herrn Streit in einen Loyalitätskonflikt zwischen ihren in Afrika lebenden Kindern und ihrer verwandtschaftlichen Gruppe einerseits und der patriarchalisch organisierten schweizerischen Familie andererseits.

Sowohl aus ethnografischen Forschungen, als auch mit Blick auf die (noch mehr oder weniger) patriarchalische Kernfamilie kann man folgern, dass der Loyalitätskonflikt zwischen der ursprünglichen und der zukünftigen Gruppe durch verwandtschaftliche Strukturen und Rituale bestenfalls in einen mehr oder weniger labilen Gleichgewichtszustand gebracht werden kann. Sowohl in traditionellen, wie auch in Gesellschaften, die sich im Umbruch befinden, geht ein permanenter Prozess des Aushandelns von Loyalitäten vor sich. Es gibt keine Garantie, dass er nicht in einen offenen Krieg zwischen den Gruppen oder in den psychischen Zusammenbruch eines Subjekts mündet. Der Austausch von Gütern kann als Funktion dieses Prozesses gesehen werden, wobei ein realer Verlust als Entlastung von einer symbolischen oder imaginären Schuld oder als Kompensation für ein Geniessen wirken kann. Frau Streits psychische Struktur stützt sich, aus dieser Perspektive gesehen, nicht (genügend) auf den Namen-des-Vaters, sondern vielmehr auf den Fluss der Gaben im Sinne eines Sinthom. Um mit den Verwandten ihres Mannes Beziehungen einzugehen, war der Austausch von Signifikanten nicht ausreichend, da ihre Signifikantenkette nicht durch einen Namen-des-Vaters verankert ist. Der Austausch von Gütern ist nicht in Fluss gekommen. Daher konnte auch kein realer Verlust in Kauf genommen werden, der vielleicht beziehungsstif- 
tend gewirkt hätte. Eine abgeschlagene Bitte, ein abgelehntes Glas Wasser, falsch verstandene Hilfsangebote haben paranoide (Hexerei-)Vorstellungen in Gang gesetzt. Die ritualisierte Form der Religionsausübung, welche Frau Streit praktiziert, konnte den «Mangel an Verlust» nicht genügend aufheben und reichte nicht aus, um als Symptom die Funktion eines Sinthom zu erfüllen.

\section{Literatur}

Evans, Dylan (2002). Wörterbuch der Lacanschen Psychoanalyse. Wien:Verlag Turia + Kant.

Freud, Sigmund (1982). Jenseits des Lustprinzips. In: Studienausgabe, Band III: 213-272. Frankfurt am Main: Fischer Verlag.

Freud, Sigmund (1999). Totem und Tabu. In: Gesammelte Werke, Band 9. Frankfurt am Main: Fischer Verlag.

Freud, Sigmund (1999). Der Mann Moses und die monotheistische Religion. In: Gesammelte Werke, Band 16: 103-246. Frankfurt am Main: Fischer Verlag. Juranville, Alain (1990). Lacan und die Philosophie. München: Boer.

Keul, Christoph (2007). Eine kurze Einführung in die Theorie der klinischen Strukturen. Riss 65-1: 11-36. Wien: Verlag Turia + Kant.

Levi-Strauss (1960). The Family. In: Harry L. Shapiro, Hrsg., Man Culture and Society. New York: Oxford University Press.

Levi-Strauss, Claude (1993). Die elementaren Strukturen der Verwandtschaft. Frankfurt am Main: Suhrkamp Verlag.

Lacan, Jacques (2005) . Le Séminaire Livre XXIII, Le Sinthom 1975-1976. Paris: Seuil.

Nasio, Juan-David (1987). Les Yeux de Laure. Paris: Flammarion.

Reed, Evelyn (1975). Woman's Evolution: from Matriarchal Clan to Patriarchal Family. New York: Pathfinder Press.

Pradelles de Latour, Henri-Charles (1991). Le crâne qui parle. Paris : E.p.e.l.

Sciacchitano, Antonello (2004). Das Unendliche und das Subjekt. Zürich: RissVerlag.

Verhaeghe, Paul (2004). On Being Normal and Other Disorders. New York: Other Press.

Verhaeghe, Paul (2009). Liebe in Zeiten der Einsamkeit. Drei Essays über Begehren und Trieb. Wien: Verlag Turia + Kant.

Widmer, Peter (2007). Paraphrenie - ein vergessenes Konzept Freuds. In: Peter Widmer, Michael Schmid, Hrsg., Psychosen: Eine Herausforderung für die Psychoanalyse. Bielefeld: Transcript Verlag, 75-101. 


\section{Anmerkungen}

1 Ich danke Micheal Schmid für seine Anregungen, die viel zur Entstehung dieses Artikels beigetragen haben.

2 Der Name und einige Angaben zu den Personen wurden verändert, um deren Anonymität zu wahren.

3 Damit ist unter Schwarzafrikanern nicht zwangsläufig eine leibliche Schwester gemeint, sondern ein Mitglied der gleichen Generation.

4 Auf die Lacanschen Konzepte Name-des-Vaters (nom/non du père) und sinthom wird weiter unten eingegangen.

5 Die Theorie der Signifikantenstruktur besagt, dass die Sprache die notwendige Voraussetzung des Unbewussten ist und dieses strukturiert ist wie eine Sprache. Die Strukturierung ergibt sich daraus, dass Signifikant (Bezeichnendes) und Signifikat (Bezeichnetes) nie ganz deckungsgleich sind. Aus dieser Diskrepanz ergeben sich die drei Dimensionen Symbolisches, Imaginäres und Reales. Das Symbolische entspricht dem Anteil des Bezeichneten, der in der Sprache erscheinen kann. Das Imaginäre steht für das Feld der Vorstellungen, Phantasien, Bilder, die durch das Symbolische evoziert werden, und das Reale ist der Anteil, der weder im Symbolischen, noch im Imaginären erscheint.

6 Ich habe mich beim «anderen» für die Kleinschreibung entschieden. Damit folge ich Lacans Unterscheidung zwischen dem kleinen und dem grossen Anderen. Der kleine andere, oder auch «a», bezieht sich, wenn der Name-des-Vaters eingesetzt ist, auf konkrete Personen, wie z. B. den Vater. Der grosse Andere, oder auch «A», stellt den symbolischen Rückhalt dar, aus dem a seine Autorität bezieht. Dieser Garant kann beispielsweise in Gesellschaften mit monotheistischen Religionen Gott sein, oder auch das Symbolische an sich, d. h. der Schatz der Sprache, in den das Kind hineingeboren wird.

$7 \quad$ Es ist zwar ein Widerspruch, sich unter dem Objekt a etwas vorzustellen, da es zur Ordnung des Realen gehört, welche sich dadurch auszeichnet, dass seine Inhalte weder symbolisierbar noch vorstellbar sind. Aber vielleicht dient es der Erklärung, es als Platzhalter gelten zu lassen, das den Platz frei hält für austauschbare Objekte, Phantasien usw.

8 Der Mangel wird konkret zunächst in der Trennung vom anderen erfahren, so dass die Symbolisierung der An- bzw. Abwesenheit des anderen einen ersten wichtigen Symbolisierungsschritt bedeutet. Siehe dazu das Fort-Da-Spiel, das Freud von seinem Enkel beschreibt (Freud ([1920] 1982: 225).

9 Mit Phantasma bezeichnet Lacan ein imaginäres Szenario, in dem das unbewusste Begehren des Subjekts in verhüllter Form dargestellt wird. Die Phantasie dient der Abwehr resp. der Verschleierung der Kastration. Das Phantasma kann erst im Lauf der Analyse in Worte gefasst werden. Der Prozess der Analyse entspricht dann der «Durchquerung des Phantasmas».

10 Diese bezieht sich auf das Paradigma des Borromäischen Knoten, mit dem sich der späte Lacan befasst hat, wo das Symbolische, Imaginäre und Reale so ineinander verschränkt sind, dass alles auseinanderfällt, wenn ein Glied der Kette gelöst wird. In der Schnittmenge aller drei Register befindet sich das Objekt a. Ein viertes Glied kann dieser Kette hinzugefügt werden. Dabei handelt es sich um das Sinthom, ein Symptom, welches das Subjekt vor der Psychose bewahrt. Lacan entwickelte dieses Modell anhand des Werkes von James Joyce, welcher nach dieser Darstellung durch sein Schreiben vor der Psychose bewahrt wurde. Er hat «sich einen Namen gemacht».

11 Siehe dazu die ethnografische Studie von Charles-Henri Pradelles de Latour bei den Bamiliké «Le crâne qui parle», wo der Autor entlang verwandtschaftlicher Beziehungen und Mitgiftregeln dieVerschränkung zwischen symbolischen Schulden resp. symbolischer Schuld und Geniessen auf der einen und symbolischen und imaginären Mitgiftforderungen auf der anderen Seite aufzeigt (Pradelles de Latour 1991). 
12 Die Orestie des Aischylos stellt eine Version eines gelungenen Überganges dar (siehe Verhaeghe 2009: 119-122). 